\title{
A Model for Interphase Chromosomes and Evaluation of Radiation Induced Aberrations
}

W.R. Holley, I.S.Mian, S.J. Park, B.Rydberg and A. Chatterjee

\author{
Lawrence Berkeley National Laboratory \\ Life Sciences Division \\ University of California \\ Berkeley, California 94720
}

Revised Version and 3 copies submitted

Number of figures: 10, Number of Tables: 1

Running title: Interphase Chromosome Model and Aberrations

Corresponding author:

Aloke Chatterjee

Lawrence Berkeley National Laboratory

Life Sciences Division 
Building 74

One Cyclotron Road

Berkeley, CA 94720

Telephone: (510) 486-5415

Fax: $\quad$ (510) 486-6949

E-mail: $\quad$ A_Chatterjee@lbl.gov

\begin{abstract}
We have developed a theoretical model for evaluating radiation induced chromosomal exchanges by explicitly taking into account interphase $\left(\mathrm{G}_{0} / \mathrm{G}_{1}\right)$ chromosome structure, nuclear organization of chromosomes, the production of double strand breaks (DSBs) and the subsequent rejoinings in a faithful or unfaithful manner. Each of the 46 chromosomes for human lymphocytes (40 chromosomes for mouse lymphocytes) is modeled as a random polymer inside a spherical volume. The chromosome spheres are packed randomly inside a spherical nucleus with an allowed overlap controlled by a parameter $\Omega$. The rejoining of DSBs is determined by a Monte Carlo procedure using a Gaussian proximity function with an interaction range parameter $\sigma$. Values of $\Omega$ and $\sigma$ have been found which yield calculated results of interchromosomal aberration frequencies that agree with a wide range of experimental data. Our preferred solution is one with an interaction range of $0.5 \mu \mathrm{m}$ coupled with a relatively small overlap parameter of $0.675 \mu \mathrm{m}$ which more or less confirms previous estimates. We have used our model with these parameter values and with resolution or detectability limits to calculate low-LET yields of translocations and dicentrics for human lymphocytes, which agree with experiments in the dose range 0.09 to $4 \mathrm{~Gy}$. Five different experimental data sets have been compared with the theoretical results. Essentially all of the experimental data falls between theoretical curves corresponding to
\end{abstract}


$1 \mathrm{Mbp}$ and $20 \mathrm{Mbp}$ resolution limits, which may reflect the fact that different investigators have different sensitivity or detectability limits. Translocation yields for mouse lymphocytes have also been calculated, and are in good agreement with experimental data from $1 \mathrm{cGy}$ to $10 \mathrm{cGy}$. There is also good agreement with recent data on complex aberrations. Our model is expected to be applicable to both low and highLET radiation and we include a sample prediction of the yield of inter-chromosomal rejoining in the dose range 0.22 Gy to $2 \mathrm{~Gy}$ of $1000 \mathrm{MeV} / \mathrm{n}$ iron particles. This dose range corresponds to average particle traversals per nucleus ranging from 1.0 to 9.12.

\section{Introduction}

Chromosome aberrations are considered to be surrogate endpoints for many deleterious biological effects such as cell killing, mutagenesis and carcinogenesis (1-3) caused by ionizing radiation. Different types of chromosome aberrations including complex chromosome rearrangements have been studied experimentally $(4,5)$ and examined using theoretical models (6-8). Accurate and reliable theoretical models can provide insights into the basic processes not accessible by direct measurements. For example, theoretical models based on mechanisms can evaluate the extent of chromosome rearrangements at low doses, a regime where current experimental techniques are not sensitive enough to provide the required information. 
Understanding interactions between lesions is important for mechanistic studies. Kellerer and Rossi (9) have employed the theory of dual action radiation to develop a model which describes interactions between lesions that can lead to cell killing. This biophysical model could explain survival curves even though it did not equate lesions with any specific type of damage such as double strand breaks. At low-LET, interactions between pairs of lesions in this model result in a linear-quadratic dose response behavior. In general, chromosome aberrations are considered to arise from interactions between two or more double strand breaks.

In investigations of the mechanisms underlying the induction of chromosome aberrations in human lymphocytes, Ballarini et al. (10) introduced a biophysical model in which 'complex lesions' (clustered DNA breaks) lead to aberrations, whereas less severe breaks are repaired. Complex lesions were defined as two or more single strand breaks (SSBs) on each strand within 30 base pairs (bp). Chromosome interphase geometry was taken into account by associating spherical domains whose volumes were proportional to the genomic DNA content of each chromosome. Their Monte Carlo calculations were specific to protons, $\alpha$ particles and carbon ions. They did not include fragments smaller than $11 \mathrm{Mbp}$ when comparing with measurements involving FISH, and $15 \mathrm{Mbp}$ when comparing with measurements involving Giemsa-staining. They obtained dose response curves for reciprocal, complex and incomplete exchanges, and each dose response curve was characterized by a parameter $f$. This parameter was defined as the fraction of chromosome free-ends initially produced which do not join with any other free-ends, leading to incomplete exchanges and/or terminal deletions. The dose response curves were calculated with $f$ arbitrarily set to $0.0,0.1,0.2$, and 0.3 . Experimental data on reciprocal exchanges were close to the theoretical results for $f=0.1$. For complex exchanges the experimental data and theoretical calculations agreed when $f=0.3$. For incomplete exchanges, the model results agreed with experiments when $f=0.2$. They 
concluded that the trends of the experimental results could be well reproduced in their model, by using different values of the parameter $f$.

In a model developed by Edwards et al. (11) for the formation of dicentrics in human lymphocytes, DNA was assumed to be randomly distributed throughout the cell nucleus. Electron tracks were simulated by a Monte Carlo approach and it was assumed that all ionizations have equal probability of inducing a DSB. This probability was adjusted to yield 50 DSBs per gray per cell, to agree with experimental results (12). The positions of energy deposition, or ionization formation in the cell nucleus was first determined, and this knowledge was then used to determine the positions of DSBs. For a known number of ionizations in each electron track, a mean number of DSBs was determined. Based on Poisson statistics, a number of ionizations was selected at random from the tracks, and DSBs placed in those positions. For rejoining of DSBs, Revell's exchange hypothesis (13) was adopted. According to this hypothesis, the initial chromosome damage consists of unstable lesions, which decay unless they interact with another lesion; exchanges are then produced as a result of pair-wise interactions between the lesions that do not undergo decay.

Sachs and collaborators have developed various theoretical models aimed at understanding the joining and misjoining of DSBs (14). They use interaction-distance concepts based on chromatin geometry, and their Monte Carlo approach CAS (chromosome aberration simulator) uses a distance algorithm for misrejoining instead of using DSB interaction sites. Many of their concepts have been incorporated in our model presented here.

Sachs et al. (15) have reviewed proximity effects in the production of chromosome aberrations by ionizing radiation. On the basis of theoretical models and experimental data, they reached the following conclusions: (i) misjoining probabilities decrease as the initial distance between DSBs increases, and almost all misrejoining 
occurs among DSBs initially separated by $<1 / 3$ of a cell nucleus diameter; (ii) chromosomes occupy irregular territories during the $G_{0} / G_{1}$ phase of the cell cycle, having dimensions also roughly $1 / 3$ of a cell nucleus diameter; (iii) proximity effects have the potential to probe how different chromosomes intertwine or move relative to each other; and (iv) incorporation of proximity effects into the classical random breakage-and-reunion model allows quantitative evaluation of yields and their interrelations for many different types of aberrations including data obtained with various FISH painting methods.

In this paper we describe a theoretical model for computing interchromosomal aberration frequencies as a function of dose. The novel aspects of our work are that we incorporate a newly developed model of the interphase structure of each chromosome packed inside a human cell nucleus and then use our previously developed track structure model to calculate specific locations in the genome of base damages and strand breaks due to ionizing radiation. Our Monte Carlo based theoretical model of the track structure of charged particles includes effects due to direct energy depositions on the DNA and indirect $\mathrm{OH}$-radical mediated effects arising from radiolysis of water in the nuclear medium of a cell (16-27). The theory incorporates detailed coordinate model descriptions of various forms of DNA from simple, linear double helices to 30 -nm chromatin fibers. The track structure model has been applied to radiolysis of pure water $(16,17)$ as well as to DNA damage studies in a simulated cellular environment (24). The theoretical results are consistent with experimental data from radiation chemistry (18) and radiation biology (27). The success of our earlier work gives us confidence that the track structure code can be employed in the calculations described here.

The DSBs are characterized in terms of their associated base damage, and this complexity can be used to determine whether a given DSB will be allowed to rejoin or 
not. Similar to other models we assume that faithful ("legitimate") and unfaithful ("illegitimate") rejoining of DSBs is influenced by "proximity" effects. In particular, we use the interaction distance model first proposed by Kellerer and Rossi (9). We calculate chromosomal aberrations by using a simple quantitative relationship to represent proximity effects. At present the model is applicable only to the $G_{0} / G_{1}$ phase of the cell.

We employ our model to determine total inter-chromosomal rejoining per cell and reciprocal translocation and dicentric formation as a function of dose for low-LET radiation, and as a function of dose and fluence for high-LET radiation. The lowest dose and the lowest fluence for which inter-chromosomal rejoinings are computed are 1cGy for low-LET radiation and an average of one particle per cell for high-LET radiation. We have also calculated the yields of complex aberrations involving three or more DSBs and two or more chromosomes. In the discussion section we describe limitations of the simple interphase chromosome model and suggest improvements.

\section{Computational Methods}

\section{Interphase Chromosome Model}

Since cells are frequently irradiated in interphase, we have concentrated, initially, on developing models for interphase chromosomes organized in a nucleus. Our initial objective is to develop models that are simple yet flexible enough to incorporate complexities in the future.

Results from a wide range of studies (27-32), can be summarized as follows:(i) the $30 \mathrm{~nm}$ chromatin fiber has a zig-zag ribbon-like structure rather than a solenoidal structure (27); (ii) large-scale chromatin organization involves the folding of $30 \mathrm{~nm}$ chromatin fibers with estimated packing ratios of hundreds to thousands; (iii) the 
chromatin is arranged in spatially separate chromosome specific territories; (iv) there is some intermingling of chromatin fibers from neighboring chromosomes which is limited to border zones of adjacent territory surfaces (32); and (v) the formation of radiation induced interchanges occurs in restricted volumes near the surfaces of adjacent territories. Based on these concepts and the random polymer model proposed by Sachs et al. (33), we have developed models of human cell nuclei containing 46 interphase chromosomes. The volume of each chromosome is assumed to be proportional to the chromosome length in base pairs. Since the chromatin model considered is a 30nm ribbon like zig-zag structure, we fold the zig-zag structure on to itself resulting in a 30nm three-dimensional fiber model similar to a "rope". This "rope" is organized as a random polymer to represent an interphase chromosome. Figure 1a shows a projected view of our model of chromosome 4. Each plotted segment represents a straight section of $30 \mathrm{~nm}$ chromatin fiber (rope) approximately $7700 \mathrm{bp}$ in length. Random steps are allowed inside the bounding spherical surface of each chromosome. A step which takes the chromatin "rope" outside the sphere is rejected and another random step is chosen and accepted only if it places the rope inside the boundary. A more rigorous approach for future extension of this work would be, for example, to use models involving semi-dilute polymer solutions with excluded volume effects. In order to test our representation of the random-walk chromosome model we compared our theoretical results for chromosome 4 with the experimental data of Trask and coworkers $(34,35)$ Using human chromosome 4 in fibroblasts cells, they measured physical separation distances between pairs of fluorescently marked probes with known lengths of DNA between them. Measurements, made during the $G_{0} / G_{1}$ phase of the cell cycle, show that on scales from $0.1 \mathrm{Mbp}$ to 1.5 $\mathrm{Mbp}$, the chromatin organization follows a random walk pattern. One of the basic characteristics of random walk, polymer behavior is that the mean-square projected two 
dimensional distance, $\left\langle\mathrm{R}^{2}\right\rangle$, between points on a DNA chain in its random conformation is linearly proportional to the genomic separation, L, between points on the chain,

$$
<\mathrm{R}^{2}>\propto \mathrm{L}
$$

Figure $1 \mathrm{~b}$ compares our theoretical model with experimental data of Yokata et al. (35) Both the experimental data and the theoretical results exhibit a biphasic $\left\langle\mathrm{R}^{2}\right\rangle$ dependence. Below $3 \mathrm{Mbp}$, results for our model chromosome agree with the experimental data including the location of the break point in the biphasic curve near 3 Mbp. Above $3 \mathrm{Mbp}$ the agreement is less satisfactory suggesting the need of a more complex chromosome model. Our results are consistent with the analysis of the same data by Sachs et al. (33) who demonstrated a biphasic nature of the slope. They argue that the reduction in slope is due to flexible chromatin loops averaging $\sim 3 \mathrm{Mbp}$, with their base points along a random walk. Although giant loops extending beyond the chromosome territory are known to exist, this feature is not a facet of our current model but could be incorporated.

\section{Interphase Nuclear Model}

Our organization of chromosomes relative to each other is based on the current state of knowledge (36-42) but will evolve as new information on chromosomes and their specific locations becomes available. Chromosome-painting studies examining regularities of chromosome arrangements have reached conclusions ranging from highly ordered structures (36) to little or no order (37). Chandley et al. (38) demonstrated that the degree of order depends on the cell type. There is some evidence for the specific positioning of chromosomes. For example, smaller chromosomes may be situated towards the interior and larger chromosomes towards the periphery of the nucleus (39), or gene-rich chromosomes such as chromosome 19 may be located in the nuclear interior and gene-poor chromosomes such as chromosome 18 located in the nuclear 
periphery (42). In the absence of definitive answers, we have positioned chromosomes within the nucleus randomly relative to each other for the calculations reported here.

Multi-color FISH studies suggest that interphase chromosomes tend to be shaped like deformed ellipsoids and that the total genomic material is constrained within a nucleus with an average diameter of $10 \mu \mathrm{m}-12 \mu \mathrm{m}$. Considering the difficulty in modeling such irregularly shaped chromosomes, for the studies reported here we have assumed that each chromosome occupies a spherical volume, and the chromosomes are contained inside a spherical nuclear volume. This assumption is more appropriate for lymphocytes than other cell types.

Since human female cells in $\mathrm{G}_{0}$ or $\mathrm{G}_{1}$ contain approximately $6.45 \times 10^{9} \mathrm{bp}$, the average interphase DNA packing density, $\rho$, in a spherical nucleus $10 \mu \mathrm{m}$ in diameter, is approximately $0.01233 \mathrm{bp} / \mathrm{nm}^{3}$. This density is within $20 \%$ of the value, $0.01528 \mathrm{bp} / \mathrm{nm}^{3}$ estimated by the fluorescence recovery after photo-bleaching (FRAP) technique (40) and is consistent with that obtained from earlier solenoidal model calculations. Using $\rho$ and the published NIH lengths of each human chromosome, the radii for "spherical" human chromosomes range from $1.0 \mu \mathrm{m}$ to about $1.7 \mu \mathrm{m}$.

Several published studies suggest that an important aspect of an interphase chromosome domain model is one that minimizes the distance between all chromosome boundaries. However, since actual chromosome boundaries are not hard spheres, allowance must be made for boundaries to intermingle. Such intermingling of chromosome boundaries leads to overlaps between chromosomes. However, the quantitative extent of such overlaps is unknown. We arrange all 46 chromosomes so they are just touching or within an assigned maximum overlap and form a nucleus approximately $6 \mu \mathrm{m}$ in radius. Our preferred model, as discussed below, has an overlap of about $7 \%$ averaged over the entire genome. This overlap percentage was determined by choosing points randomly inside the cell nucleus and taking the ratio of points inside 
two or more chromosomes to points inside at least one chromosome. Points outside all the chromosomes were not counted in assessing this percentage.

Based on the considerations detailed above, a Fortran program POSITION was developed to arrange 46 spherical human chromosomes randomly within a spherical nucleus. The code employs the following parameters: (1) $r_{\max }$, the radius of the sphere containing all the chromosomes (2) $\Omega$, the maximum overlap allowed among domains, and (3) $\Gamma$, the maximum gap allowed between domain surfaces.

The packing procedure starts by randomly choosing a chromosome from the possible 46 and placing it at a randomly chosen position in the nucleus. From the remaining chromosomes, a new chromosome is randomly chosen and is placed randomly within the nucleus. The surface-to-surface distance between the new chromosome and all previously positioned chromosomes is calculated. The test position is accepted if the overlap with all other volumes is less than the maximum overlap $\Omega$, and the gap between the test volume and at least one other previously positioned chromosome volume is less than the gap parameter $\Gamma$. If either of these criteria fails, a new chromosome is chosen. The procedure continues until all 46-chromosome domains are positioned. In order to randomize the chromosome packing for different trials with radiation tracks, the whole procedure can be repeated beginning with the placement of the first chromosome. Rather than this ad hoc packing procedure a preferred method for future extensions of this work would be to model the whole nucleus by polymer methods with excluded volume effects as suggested for individual chromosomes.

Figure 2 shows a typical example of chromosomes packed within a circumscribing radius of $6.5 \mu \mathrm{m}$. This packing results in an average overlap of about $7 \%$ for the entire genome. Note that $r_{\max }(6.5 \mu \mathrm{m}$ in this example) is a limiting radius used in packing chromosomes inside a nuclear volume. The average or effective radius of the 
model nucleus is appreciable smaller if measured from the outer chromosomes surfaces, and is closer to $5.5 \mu \mathrm{m}$ in this example.

\section{Mechanistic Models for Initial DNA Damage at the Molecular Level}

Our existing track structure code is based on the microscopic distribution of energy depositions around the trajectory (the physical stage) of a charged particle track, and includes the ensuing radiation chemistry that evolves due to the radiolysis of water contained in a cellular environment. Besides primary electron and heavy charged particle tracks, secondary and tertiary tracks are also considered. Since low energy electron tracks (below $2000 \mathrm{eV}$ ) undergo extensive large angle multiple scattering (24), their energy deposition distributions are evaluated appropriately. High-energy electron tracks are considered to deposit energy along a linear path. We have successfully used these methods for analyzing both radiolysis of water (18) and also DNA in solution $(21,22)$. We calculate the contributions due to indirect (radical) mechanisms and direct mechanisms of damage as summarized below (24). Irrespective of the structural form of DNA (linear molecule, beads-on-a-string or chromatin), our code explicitly considers the locations of nucleotides $\mathrm{A}, \mathrm{T}, \mathrm{G}$ and $\mathrm{C}$ in a three-dimensional coordinate frame of reference and provides details of which bases and which sugars are damaged. It will be straightforward in the future to include specific DNA sequences in our model.

\section{Indirect Damage}

Tracks react with water molecules and create radical products, primarily $\bullet \mathrm{OH}$, $\bullet \mathrm{H}, \mathrm{e}^{-}{ }_{\text {aq }}$ and $\mathrm{H}_{3} \mathrm{O}^{+}$. These radicals can react with each other, with a DNA fiber or with a scavenger representing the proteins, RNAs, and other molecules near the fiber. Using a Monte Carlo simulation procedure, the diffusion of each radical is followed in space and time. If two radicals come within a certain reaction distance of each other 
(Smoluchowski radius), they are assumed to react, forming a molecular product, and the radical diffusion path is no longer followed. If a surviving radical diffuses within the critical radius of a DNA site, it reacts with the particular site. The respective critical radii or reaction radii (determined by measured rate constants) for $\bullet 0 \mathrm{H}$ and the various sites (21) are: sugar, 1.0 $\AA$; adenine (A), $3.6 \AA$ thymine (T), $3.6 \AA$; guanine $(\mathrm{G}), 5.2 \AA$; and cytosine $(\mathrm{C}), 3.6 \AA$ A.; In this manner, we calculate individual damages distributed along the genome and their respective yields, including clusters of damage sites, i. e. within $20 \mathrm{bp}$ of each other. In our model, $\bullet \mathrm{OH}$ attacks on sugars lead to the formation of SSBs but those on bases do not lead to strand breaks.

\section{Direct Damage}

In the Monte Carlo computation, we consider a collection of tracks impinging on a segment of chromatin fiber. The coordinates of a track, its lateral extension, and the DNA model are used to determine which sugar-phosphate molecular groups lie within the track. The energy deposition path of a secondary electron is represented as uniformly occupying a cylindrical volume of length equal to the rms range of the electron and radius $\left(r_{\text {eff }}\right)$ roughly that of a typical spur $(\sim 20 \AA)$. For example, for an electron with an rms residual range $X$, we use a simplified version of the Bragg rule (20) to estimate the average energy deposited on a DNA molecular group:

$$
\Delta \mathrm{E}=\left\langle\frac{d E}{d X}\right\rangle \frac{\sum Z_{i}}{2 \pi r_{e f f}^{2} n_{e 1}}
$$

Where $\langle\mathrm{dE} / \mathrm{dX}\rangle$ is the effective LET in water for electrons of energy $\mathrm{E}$ and rms residual range $X, n_{\mathrm{e} 1}$ is the electron density of water, $Z_{\mathrm{i}}$ is the atomic number of the $i$ th atom and the summation runs over all atoms in the molecular group. Energy is actually deposited on a DNA molecule in a random or stochastic fashion through electronic excitation. Because detailed cross-sections for DNA excitation processes are not known, we 
represent them by an average process characterized by a mean energy transfer, $\langle E\rangle$, of $29.9 \mathrm{eV}$ determined from the DNA oscillator strength measurements of Inagaki (43). We assume that all such energy transfers lead to ionizations.

For each incident particle, we calculate the probability of ionization, $\mathrm{P}$, for every reaction site within the track cylinder. We generate an "event" by choosing a random number, $\mathrm{R} \#$, for each of these sites. If $\mathrm{R} \#<\mathrm{P}$ for a particular site, we consider that an ionizing reaction has occurred signaling the production of a DNA strand break (sugar damage) or a base damage. Two SSBs (whether direct or indirect) on opposite strands separated by $<10 \mathrm{bp}$ form a DSB.

Simulation of Tracks Through a Cell Nucleus and Damage Assessment

A Monte Carlo simulation procedure is used to study the interaction of charged particle tracks with the interphase nucleus. For a given dose D, in Gray, the average number $\langle\mathrm{N}\rangle$ of incident particles is determined using the relationship

$$
<\mathrm{N}>=6.42 \quad \frac{D ? \pi R^{2}}{L E T}
$$

where $\mathrm{R}$ is radius of nucleus in $\mu \mathrm{m}$ and LET is in $\mathrm{kev} / \mu \mathrm{m}$.

For a given trial, $\mathrm{N}$ tracks chosen from a Poisson distribution with average $\langle\mathrm{N}\rangle$ are generated either randomly and isotropically throughout the nucleus, or as a set of randomly spaced parallel tracks in a randomly chosen direction to simulate exposures made with an accelerator beam. In our interphase chromosome model, each chromatin fiber in the random polymer is identified both in terms of its respective number in a specific chromosome and its position coordinates. Each of the bases and sugar molecules in a given fiber is identified with co-ordinates in three dimensions. The locations of features such as centromeres and telomeres are available for each chromosome.

For each simulated track, fiber segments that lie within a specified distance from the track are considered for further computational procedures to assess damage by 
indirect and direct effects. The distance is the sum of the range of a 2 kev electron in liquid water and the characteristic $\bullet \mathrm{OH}$ diffusion distance of $4 \mathrm{~nm}$. This criterion is based on "local" energy deposition by a track, and has proven useful previously for the assessment of damages to DNA sites $(16,24)$. Secondary electrons above $2 \mathrm{keV}$ are treated as separate tracks and their contribution to damage is evaluated separately. Once a track and a chromatin fiber or fibers are identified as potentially able to interact, damages to the DNA bases and sugars are calculated. The trial number, track number, chromosome number, fiber number and index position within the fiber are recorded for each damage site. The output from this process allows us to identify base damages, SSBs and DSBs. In this manner we generate a complete listing of all DSBs inside a cell nucleus, including their chromosome specific and geometric positions. Such information is then used to calculate chromosome aberrations.

\section{Estimation of Chromosome Aberration Frequencies}

The starting point for estimating chromosome aberrations is the positions of DSBs and the distances between pairs of such breaks produced by tracks of charged particles. The two ends of a break can be either rejoined together (faithful rejoining) with perhaps a local error of a few base pairs, or the ends can be independently joined with available ends from other breaks (unfaithful rejoining) in a breakage-and-reunion model leading to aberrations. Unfaithful pairings can occur within a chromosome or involve two or more chromosomes. Repair protein complexes are involved in correct as well as incorrect rejoining processes, and, as in other published models, we assume that these complexes are unable to distinguish between the origins of the broken ends. Currently, we require at least two DSBs produced by radiation to generate a chromosomal rearrangement and use an interaction distance model characterized by the initial separation between DSBs.

The interaction distance model (9) is based on proximity effects. Similar models have been extensively applied to measurements related to soft X-rays $(44,45)$ and in the 
determination of RBE-LET relationships (46). Proximity effects are modeled by assigning specific probabilities that are some function of the distance between the DSB free ends. We use a simple Gaussian proximity function given by

$$
\text { Prob } \propto \exp \left(-\mathrm{d}^{2} / 2 \sigma^{2}\right)
$$

where $\mathrm{d}$ is the initial distance between any two ends and the range parameter $\sigma$ is the standard deviation of the range of interaction of two ends. In our model, the value of $\sigma$ needed to fit the inter-chromosomal aberration data depends on the amount of overlap between chromosomes, $\Omega$, and cannot be determined uniquely since $\Omega$ is not well known at the present time. In these calculations we use the experimental observations $(47,48)$ that, at high doses, the faithful rejoining fraction reaches a plateau value of about $55 \%$ of the initial number of DSBs. Thus we choose $55 \%$ of the breaks at random and assign them to be rejoined faithfully. The rejoining fidelity of the remaining $45 \%$ is decided based on the proximity function. A similar separation of DSBs into two types for modeling rejoining was used by Radevoyevitch et al. ( 49,50). The rejoining algorithm proceeds as follows: (1) The relative probabilities of rejoining between all unjoined ends are calculated using the Gaussian expression above. (2) The probabilities are normalized to unity by dividing by the sum of all probabilities. (3) A rejoining interaction between two ends is selected by means of a random number uniformly distributed in the range 0.0 to 1.0. (4) The two joined ends are removed from the set of unjoined ends and the procedure is repeated until all ends with non-zero rejoining probabilities have joined. Using this procedure, occasionally, isolated or "orphan" ends will remain unjoined if a limiting interaction range has been introduced into the proximity function definition. For an interaction range limit of $2 \mu \mathrm{m}$, this fraction, in our calculations, is generally less than $1 \%$ of the rejoining candidate ends and has been neglected in the analysis. 
Figure 3 illustrates the simplest configurations resulting from the rejoining calculation. Shown are examples of formation of a complete translocation, dicentric, acentric ring (or deletion), inversion, centric ring and simple complex rearrangement (insertion). Of course there are a multitude of other possible configurations, especially complex events that occur at very low frequency. In our model the probability of two ends joining depends on the initial separation of the breaks. Therefore it is clear that the probability of a simple reciprocal translocation formation (Fig. 3a) is the same as the probability of dicentric formation (Fig. 3b). If one neglects complex rearrangements the number of reciprocal translocation or dicentric-acentric fragment events will each equal $1 / 4$ the total number of inter-chromosomal end joins. For purposes of this paper we have used this simple approximation. A detailed analysis, which involves identifying each of the translocation and dicentric events in the human cell calculations, shows that this approximation is good to better than $1 \%$ at doses up to $1 \mathrm{~Gy}$. At high doses the number of complex rearrangements increases, but the approximation overestimates the yield of translocations and dicentrics by less than $10 \%$ at $5 \mathrm{~Gy}$.

In the version of the rejoining algorithm presented here, rejoining of DSB ends is followed to completion, and, therefore, terminal deletions have not been modeled. Deletions appear as acentric (Fig. 3c) and centric (Fig. 3e) rings. In the future, terminal deletions can be introduced into the model in several possible ways. 1. Certain DSBs could be selected as non-joining because of complexity (e.g. number of associated base damages). 2. Upper limits could be placed on the range of allowed rejoining interactions; i.e, The proximity function could be set to zero for separation distances greater than 1.5 or $2 \mu \mathrm{m}$ leading to occurrence of isolated or "orphan" ends which could be identified with terminal deletions. 3. The rejoining could be arbitrarily terminated after a fraction, determined by terminal deletion data, of the ends have rejoined. The first 
two procedures would be preferred over the ad hoc third method as more easily related to mechanisms.

The error bars in our calculations are determined by Poison statistics and depend upon the number of trials. By choosing a large number of trials we could make the error bars as small as we want, but no such attempt was made in view of the scatter in the experimental data.

\section{Results}

Yields and Distributions of DSBs and Inter-Chromosomal Rejoinings

We have calculated DSB distributions and yields for different radiation qualities at several dose levels. For $1 \mathrm{~Gy}$ of low-LET electrons (similar to X-ray irradiation), the breaks are approximately randomly distributed throughout the nucleus ( Fig. 4a) . For 1 Gy irradiation by a beam of $1 \mathrm{MeV} / \mathrm{n}$ helium particles, the breaks are well correlated with parallel tracks( Fig. 4b). Since the incident energy is very low, the associated secondary electron energies are also very low and their contributions in producing DSBs cannot be separated spatially from the primary tracks on a scale representing the nucleus, chromatin and chromosomes. The number of DSBs produced by He in this trial, 108, is appreciably larger than the 46 DSBs in the electron example (Fig. 4a). Our theoretical

yields, averaged over many trials, are slightly higher (15\%) than typical experimental measurements for helium ions between $0.75 \mathrm{MeV} / \mathrm{n}$ and $1.75 \mathrm{MeV} / \mathrm{n}(51)$. In the case of the $1000 \mathrm{MeV} / \mathrm{n}$ iron particles (Fig.4c) the associated high energy $\delta$-rays create their own tracks which can produce DSBs distinct and separate from the primary iron tracks. Thus, some open circles lie along the parallel primary tracks and others appear to be distributed randomly due to the high-energy $\delta$-rays. 
Based on the specific location of breaks within the cell nucleus for each trial and the rejoining algorithm, we have estimated the frequencies of chromosome aberrations as a function of dose. Figure 5 represents our calculated yields of total inter-chromosomal rejoinings per cell as a function of dose in the range from 0 to 4 Gy for low-LET electrons. Three values of the range parameter $(\sigma)$ were used in these calculations $(400$, 500 and $600 \mathrm{~nm}$ ). The chromosome maximum overlap parameter, $\Omega$, was set to 0.675 $\mu \mathrm{m}$ and the gap parameter, $\mathrm{T}$, to $0.0 \mu \mathrm{m}$ for the calculations. The yield of interchromosomal exchanges (rejoins) in this dose range has a linear-quadratic behavior. Figure 5 includes all inter-chromosomal exchanges, including those that involve DNA segments that are too small to be detected by existing cytogenetic techniques. Figure 6 shows inter-chromosomal rejoinings for the $\sigma=0.5 \mu \mathrm{m}$ case with resolution limits of $\geq 1$ bp (all unfaithful rejoinings), $\geq 1 \mathrm{Mbp}, \geq 10 \mathrm{Mbp}$ and $\geq 20 \mathrm{Mbp}$. The differences between the curves for $1 \mathrm{bp}$ resolution and $1 \mathrm{Mbp}$ resolution are negligible demonstrating that, at low LET, there are very few interchanges involving DNA segments smaller than $1 \mathrm{Mbp}$. The differences between $1 \mathrm{Mbp}$ and $10 \mathrm{Mbp}$, and also between $10 \mathrm{Mbp}$ and $20 \mathrm{Mbp}$ are more substantial. About $30 \%$ of the unfaithful rejoinings involve DNA segments smaller than $20 \mathrm{Mbp}$ and this fraction appears to be independent of dose in the dose range we have studied. It should be noted that different experimental techniques have different resolution limits. Knowledge of these limits is essential before a meaningful comparison with the theoretical results can be made. However, theoretical estimates with different resolution limits can be helpful in careful analysis of experimental data.

As discussed in the previous section, in our model, the theoretical translocation and dicentric frequencies should be equal, irrespective of the location of the centromeres at any dose. Experimentally, Loucas and Cornforth (5) have determined the relative proportion of these two types of aberrations as approximately 1:1. For certain other calculations, such as centric ring production, one must include, explicitly, the 
locations of the centromeres. We have done this in preliminary studies using the human cell model. As an example we have taken into account the precise positions of centromeres in calculating translocations and dicentrics production at $2 \mathrm{~Gy}$. The resulting numbers of translocations dicentrics were 24 and 21 , respectively, well within the statistical errors of each other. Centromere locations can easily be included in our mouse model as well. However, before calculating detailed centric ring and other intrachromosomal aberration frequencies, certain improvements in the current interphase chromosome model are needed as explained in the discussion section.

The calculated total inter-chromosomal rejoining events included in our calculation approximately equals the sum of simple translocation and dicentric events. Complex events, defined as involving three or more DSBs on two or more chromosomes, correspond to a small fraction of these exchanges and are discussed later. Figure 7 shows our calculated reciprocal translocation (or dicentric) frequencies and standard errors based on Poisson statistics in the dose range up to $4 \mathrm{~Gy}$ for two resolution limits, $1 \mathrm{Mbp}$ and $20 \mathrm{Mbp}$. As discussed in the previous section we use the approximation that the translocation (or dicentric) frequency is simply one quarter the number of interchromosomal rejoinings per cell. Regression curves have also been plotted using the calculated points and linear quadratic expressions to obtain the best fit. The fitted expression for $1 \mathrm{Mbp}$ resolution is $0.1155 \mathrm{D}+0.06152 \mathrm{D}^{2}$ and for $20 \mathrm{Mbp}$ resolution is $0.07072 \mathrm{D}+.045105 \mathrm{D}^{2}$. The theoretical results are compared with five different sets of experimental data. Since measurements of Lucas et al. (52) include only translocations involving the $\mathrm{p}$ arm of chromosome 1 with any other remaining chromosome and our $\sigma$ calculations include all translocations, the experimental data were adjusted to correspond to total translocation frequencies for all chromosomes. Using human lymphocytes in $\mathrm{G}_{0}$ and irradiating with X-rays from 0.5 to 4 Gy, Finnon et al.( 53 ) measured chromosomal 
aberrations. We have plotted their data on translocation frequencies in Figure 7. Since the yields of dicentrics and translocations are equal in our model, we have also plotted yields of dicentrics from Lloyd et al. (54) and Edwards (55) in the same figure. From the figure it can be seen that essentially all the data from these five experiments are bounded by the two theoretical curves having resolution limits of $1 \mathrm{Mbp}$ and $20 \mathrm{Mbp}$. The theoretical calculations for range parameter $\sigma=0.5 \mu \mathrm{m}$ and overlap parameter $\Omega=$ $0.675 \mu \mathrm{m}$ agree well with the experimental results within these two limits.

As a stringent test of the applicability of the model we have also compared our calculated results with experimental values in the very low dose region. Tucker et al. (56) performed whole body irradiation of mice with 21, 42 and 63 fractions, and measured yields per fraction of chromosome aberrations in lymphocytes at doses per fraction as low as $1 \mathrm{cGy}$. In order to compare our calculations with this data we developed models of mouse nuclei (40 chromosomes) using similar size and overlap parameters as in the human nuclei models. Figure 8 compares the experimental data with calculated results for three different values of the range parameter: $\sigma=0.3 \mu \mathrm{m}, 0.5$ $\mu \mathrm{m}$ and $0.6 \mu \mathrm{m}$. As at the higher doses for human, an overlap parameter $\Omega=0.675 \mu \mathrm{m}$ with $\sigma=0.5 \mu \mathrm{m}$ gives good agreement with the experimental data for yields of translocations per 100 cells at doses ranging from $1 \mathrm{cGy}$ to $8 \mathrm{cGy}$. Because Tucker et al. (56) count each of the two color junctions in a reciprocal translocation separately, our calculated results are simply the total yield of inter-chromosomal rejoinings divided by two to account for dicentric-acentric fragment production. Calculations (results not shown) done with the human nucleus model in this dose range are in substantial agreement with the theoretical results and experimental data for mouse cells. 
Variation in Range Parameter as a Function of Overlap Parameter

Given our current knowledge of the structure of interphase chromosomes and their localizations in a cell nucleus relative to each other, the extent of overlap between them is not well known. Two color, chromosome painting, laser-confocal, serial sections and 3D-reconstruction of neighboring and overlapping chromosome territories suggest that intermingling of chromatin fibres is limited to peripheral regions of adjacent territory surfaces $(41,42)$. However, there is no quantitative information on this overlap. It is evident that the extent of overlap, $\Omega$, and the range parameter $\sigma$ are interrelated. We have studied this relationship as it pertains to the present model and prescribed what we consider to be an acceptable level of overlap in organizing the various chromosomes. So far all the results presented on inter-chromosomal frequencies correspond to an allowed overlap between neighboring chromosomes of $\Omega=0.675 \mu \mathrm{m}$. For this overlap, $\sigma=0.5 \mu \mathrm{m}$ gives good agreement between the experimental and theoretical translocation frequencies for both mouse and human. We studied two more maximum allowable overlaps $0.3 \mu \mathrm{m}$ and $1 \mu \mathrm{m}$.

For $\Omega=0.3 \mu \mathrm{m}, \sigma=0.525 \mu \mathrm{m}$ gives the best agreement with the experimental translocation frequencies between $0 \mathrm{~Gy}$ and $5 \mathrm{~Gy}$. Similarly, for $\Omega=1 \mu \mathrm{m}$ the best agreement was obtained for $\sigma=0.4 \mu \mathrm{m}$. The results are summarized in Table I. In the present model, a three fold change in the maximum overlap limit $\Omega$ is accompanied by a 1.3 fold change in the corresponding range parameter $\sigma$. These results suggest that the range parameter is relatively insensitive to the precise value of the overlap parameter, and most of our calculations have been made with the intermediate set of parameters : $\Omega=$ $0.675 \mu \mathrm{m}, \sigma=0.5 \mu \mathrm{m}$.

\section{Complex Aberrations}

Several studies (4) have demonstrated complex interactions between three or more DSBs distributed among two or more chromosomes using combinatorial multi-fluor 
fluorescence in situ hybridization (mFISH). Significant frequencies of these types of aberrations were found in human lymphocytes for exposure with $\gamma$-rays at doses between 2 and 4 Gy. Roughly half of the cells at 4 Gy contained at least one complex exchange that required anywhere from 3 to 11 initial chromosomal breaks. At this dose, more than $40 \%$ of gross cytogenetic damage, as measured by the total number of exchange break points, was complex.

Our model provides complete details of all interchanges, such as chromosome number and locations on chromosomes. At higher doses (>2Gy), we find a substantial number of exchanges involving three or more breaks and two or more chromosomes. Figure $3 f$ shows the simplest example of a complex interaction. The calculated yields of the fraction of cells that have at least one complex interchange as a function of dose between 0 to 4 Gy are shown in Figure 9. The data of Loucas and Cornforth (5), for the most part, lie between the theoretical results for resolution limits of $10 \mathrm{Mbp}$ and $20 \mathrm{Mbp}$. Although the experimental resolution limit is uncertain, it is generally believed to fall between $10 \mathrm{Mbp}$ and $20 \mathrm{Mbp}$.

The calculations of complex rearrangements presented in this section used an overlap parameter $\Omega=0.675 \mu \mathrm{m}$ and a range parameter $\sigma=0.5 \mu \mathrm{m}$, values which also gave good agreement with translocation measurements in the 0-4 Gy and 0-10 cGy dose ranges. Thus no further adjustment was necessary to agree with the data from complex exchanges. The fraction of cells showing at least one complex exchange at 4 Gy was calculated to be $43 \%$ and is consistent with the $44 \%$ value reported by Loucas and Cornforth (5).

\section{Prediction of the Model at high LET}

The model has also been used to calculate the inter-chromosomal rejoinings per cell for $1000 \mathrm{MeV} / \mathrm{n}$ iron particles. Future high-LET measurements of translocation 
frequencies as a function of fluence would be useful in order to test the predictions of the model. Although it is possible that the rejoining mechanisms or the effective rejoining parameters are different for high and low-LET radiation (e.g., the increased complexity of damage in the vicinity of high-LET DSBs could influence repair times and allow broken ends to diffuse further, thus increasing the effective range of interaction) we have made the simplifying assumption, for the high-LET calculations, that rejoining will be governed by the same proximity function with the same parameters $(\sigma=0.5 \mu \mathrm{m}$ and $\Omega=$ $0.675 \mu \mathrm{m})$ as for low-LET. The fact that the limiting values of the fractions of faithful and unfaithful rejoining at very high doses (48) are the same for high and low-LET suggests that the same rejoining mechanisms may be involved.

The LET of an iron nucleus is about $150 \mathrm{keV} / \mu \mathrm{m}$ for an incident energy of 1000 $\mathrm{MeV} / \mathrm{n}$. The average total number of DSBs for these ions for $1 \mathrm{~Gy}$ of radiation is 80 . These breaks are produced by the primary particle as well as $\delta$-rays ( Fig. 4c). Figure 10 shows the theoretical inter-chromosomal rejoining frequency as a function of dose together with the average fluence through the cell nucleus at each dose point. Our theoretical results for high-LET heavy ions show a linear dose dependence instead of the linear-quadratic dose dependence evident for low-LET radiation. The linear dependence suggests that (a) single tracks produce enough DSBs to cause misrejoining and (b) at the doses studied here, the number of tracks is low enough ( $<10$ tracks/ nucleus at 2 Gy) that breaks from different tracks are unlikely to be close enough to each other to interact. Although no measurements of translocation frequencies have been made, a linear dose dependence for $1000 \mathrm{MeV} / \mathrm{n}$ iron particles has been reported by Brooks et al.( 57) for chromatid type aberrations. 


\section{Discussion}

The primary goal of this study was to use a simple model representing interphase chromosomes to evaluate, quantitatively, chromosomal aberrations induced by high and low doses of ionizing radiation. The results indicate that this simplified model is able to provide yields of aberration frequencies that are in reasonable agreement with the available experimental data. In addition to the interphase chromosome model, the overall procedure incorporates elements of track structure to simulate production of DSBs and an algorithm for faithful and unfaithful rejoining based on the proximity of DSBs to each other. Plausible values of the chromosome overlap parameter and the DSB interaction range parameter give good agreement with the experimental data for both simple and complex aberrations. The extent to which chromosomes actually overlap with each other is not well known.

In spite of the above success, there are deficiencies in the interphase chromosome model. Figure $1 \mathrm{~b}$ shows that the computed mean square distances between fluorescent markers for genomic separations up to $3 \mathrm{Mbp}$, agree very well with in situ hybridization measurements. However, for separations larger than $3 \mathrm{Mbp}$, the agreement is poor indicating a need for improvement in the model. Although there is quantitative disagreement, both the experimental data and the theoretical computation show biphasic behavior in the slope of the mean square projected separations versus genomic distance.

Currently we are evaluating the frequencies of intra-chromosomal exchanges as a function of dose. This evaluation will provide another test of the adequacy of the interphase chromosome model presented here. Our initial results indicate that, in general, total intra-chromosomal exchange frequencies are about three times greater than total inter-chromosomal exchange frequencies. In the case of centric-ring formation, we find a 
major discrepancy between our calculated results and available experimental data. According to Simpson and Savage (58), the sum of the yields of translocations and dicentrics at $6 \mathrm{~Gy}$ of X-rays is more than twenty times greater than that of centric rings. Conversely, the yields of centric rings from our calculations are two to three times larger than the sum of translocations and dicentrics. Such a large difference between theory and experiment suggests that substantial modifications need to be made, at least for centric ring calculations. A likely source of the error in our model appears to be the assumption that chromosomes are organized as completely random polymers within their territories. Such a scheme necessarily completely mixes together DNA from both the $\mathrm{p}$ and $\mathrm{q}$ arms of a chromosome and leads to relatively large probabilities of centric-ring formation.

One of the modifications we are considering at the present time is the separation of chromosome arms. Spatial separation between $\mathrm{p}$ and $\mathrm{q}$ arms in interphase chromosomes has been observed in simulation and experimental studies by Munkel et. al.( 30) using confocal laser scanning microscopy. To improve our present interphase chromosome model, we plan to adopt a slightly modified version of their procedures. By separating the $\mathrm{p}$ and the $\mathrm{q}$ arms in space, we expect to greatly reduce the calculated frequencies of centric rings. The yields of total intra- and inter-chromosomal exchanges should not be appreciably affected, however, as long as overall chromosomal volumes, shapes and overlaps remain unchanged. These modifications may also improve agreement between the computed mean square separation of chromosome specific markers and fluorescent in situ hybridization measurements for large genomic separations.

The interphase chromosome structure model should eventually incorporate the existence of loops fixed at matrix attachment sites. However, there does not yet appear to be a consensus in the literature in terms of the actual sizes of loops or their organization. Based on the measurements of the distance between points on the same chromosomes, 
Yokota et al.( 35) proposed that there are two organizational levels at scales greater than 100kbp. At the first level, they suggest that chromatin is in large loops several Mbp in size and within each loop chromatin is folded randomly. At the second level, they suggest that the specific loop-attachment sites are arranged to form a supple, backbone like structure, which also shows characteristic random walk behavior. In contrast, simulations and measurements by Munkel et al.(30) suggest that chromatin is folded into $120 \mathrm{kbp}$ size loops which are assembled into rosette-like subcompartments. These subcompartments are connected by small segments of chromatin. The number and size of subcompartments correspond with chromosome bands in early prophase. We are currently developing chromosome models incorporating these ( and other) loop concepts, the results of which will be reported elsewhere.

In conclusion we have presented a detailed model which accurately describes a wide range of radiation induced inter-chromosomal aberrations. The model combines our well-described track structure model with simple interphase models of both human and mouse nuclei and a DSB free- end- rejoining algorithm based on spatial proximity. This model can be used to predict the production of inter-chromosomal aberrations by any incident particle at any energy.

\section{ACKNOWLEDGMENTS}

This research was funded by the Low Dose Radiation Research Program, Biological and Environmental Research (BER), U. S. Department of Energy, under contract no. DEAC03-76SF00098. Additional support was provided by a NASA Specialized Center of Research and Training (NSCORT). The authors thank Ryan Alexander for developing the POSITION algorithm for placing chromosomes inside a nucleus and for many helpful

discussions, and Bipasha Mukherjee for many helpful suggestions and assistance in preparation of this manuscript. 


\section{References}

1. J.S. Bedford, J.B. Mitchell, H.G. Griggs, and M.A. Bender, Radiation induced cellular reproductive death and chromosome aberrations. Radiat. Res. 76, 573-586 (1978).

2. A.J. Grosovsky, K.K Parks, C.R. Giver, and S.L. Nelson. Clonal analysis of delayed karyotypic abnormalities and gene mutaions in radiation-induced genetic instability. Mol. Cell. Biol. 16, 6252-6262 (1996).

3. J.W. Gray, D. Pinkel, Molecular cytogenetics in human cancer diagnosis. Cancer 69, 1536-1542 (1992).

4. M. N. Cornforth, Analyzing radiation-induced complex chromosome rearrangements by combinatorial painting. Radiat. Res. 155, 643-659 (2001).

5. B. D. Loucas and M. N. Cornforth, Complex chromosome exchanges induced by gamma rays in human lymphocytes: An mFISH study. Radiat. Res. 155, 660-671 (2001).

6. R.K. Sachs, A.M. Chen, P.J. Simpson, L.R. Hlatky, P. Hahnfeldts and J.R.K. Savage, Clustering of radiation-produced breaks along chromosomes: Modeling the effects on chromosome aberrations. Int. J. Radiat. Biol. 75, 657-672 (1999).

7. R.K. Sachs, D. J. Brenner, A.M. Chen, P. Hahnfeldt, and L.R. Hlatky, Intra-arm and Inter-arm chromosome interchanges: tools for probing chromatin geometry and Dynamics, Radiat. Res. 148,330-339 (1997).

8. D.J. Brenner and R.K. Sachs, Commentary: Chromosome 'fingerprints' of prior exposure to densely ionizing radiation. Radiat.Res. 140, 134-142 (1994).

9. A.M. Kellerer and H.H. Rossi, A generalized formulation of dual radiation action. Radiat. Res. 75, $471-488$ (1978).

10. F. Ballarini, M. Merzagora, F. Monforth, M. Durante, G. Gialenella, G.F. Grossi, M. Pugliese and A.Oltolenghi, Chromosome aberrations induced by light ions: Monte Carlo simulation based on mechanistic model. Int. J. Radiat. Biol. 75, 35-46 (1999).

11. A.A. Edwards, V.V. Moiseenko and H. Nikjoo, On the mechanism of the formation of chromosomal aberrations by ionizing radiation. Rad. Environ. Biophy. 35, 25-30 (1996).

12. J.F. Ward, The yields of DNA double-strand breaks produced intracellularly by ionizing radiation: a review. Int. J. Radiat. Biol. 57, 1141-1150 (1990).

13. S.H. Revell, The breakage-and-reunion theory and the exchange theory for chromosomal aberrations induced by ionizing radiation: a short history. Advances in Radiat. Bio. 4, 367-416 (1974). 
14. R. K.Sachs, Levy, D., Chen, A. M., Simpson, P. J., Cornforth, M. N., Ingerman, E. A., Hagnfeldt, P., and Hlatky, L.R., Random breakage-and- reunion chromosome aberration formation model: An interaction-distance implementation based on chromatin geometry. Int. J. Radiat.Biol. 76, 1579-1588 (2000).

15. R.K. Sachs, A.M. Chen and D.J. Brenner, Review: proximity effects in the production of chromosome aberrations by ionizing radiation. Int. J. Radiat. Biol. 71, 1-19 (1997).

16. J. L. Magee and A. Chatterjee, Track reactions of radiation chemistry. Kinetics of Non homogeneous Processes. (Gordon R. Freeman, Ed.) John Wiley and Sons, Inc. pp. 171-213 (1987).

17. J.L. Magee and A. Chatterjee, Radiation chemistry of heavy particle tracks. 1. General considrations. J. Phys. Chem. 84, 3529-3536 (1980).

18. A. Chatterjee and J.L. Magee, Radiation chemistry of heavy particle tracks. 2. Fricke dosimeter system. J. Phys. Chem. 84,3537-3542 (1980).

19. A. Chatterjee and W. R. Holley, Energetic electron tracks and DNA strand breaks. Nucl. Tracks Radiat. Meas. 16, 127-133 (1989).

20. W.R. Holley, A. Chatterjee and J. L. Magee, Production of DNA strand breaks by direct effects of heavy charged particles. Radiat. Res. 121, 161-168 (1990).

21. A. Chatterjee and W. R. Holley, A general theory of strand break production by direct and indirect effects. Rad. Prot. Dosimetry 31, 241-247 ( 1990 ).

22. A. Chatterjee and W.R. Holley, Energy deposition mechanisms and biochemical aspects of DNA strand breaks by ionizing radiation. Int. J. Quantum Chem. 39, 709727 (1991).

23. A. Chatterjee and W. R. Holley, Biochemical mechanisms and clusters of damage for high-LET radiation. Adv. Space. Res. 12, 33-43 (1992).

24. A. Chatterjee and W. R. Holley, Computer simulation of initial events in the biochemical mechanisms of DNA damage. Advances in Radiation Biology (J. T. Lett and W. K. Sinclair, Eds.) Academic Press, San Diego. 17, 181-226 (1987).

25. W.R. Holley and A. Chatterjee, Clusters of DNA damage induced by ionizing radiation: Formation of short DNA fragments. I. Theoretical Modeling. Radiat. Res. 145, 188-199 (1996).

26. B. Rydberg, Clusters of DNA damage induced by ionizing radiation: formation of short DNA fragments. II . Experimental detection. Radiat. Res. 145, 200-209 ( 1996).

27. B. Rydberg, W.R. Holley, I.S. Mian and A. Chatterjee, Chromatin Conformation in living cells: support for a zig-zag model of the $30 \mathrm{~nm}$. chromatin fiber. J. Molec. Biol. 284, 71-84 (1998).

28. T. Cremer, A. Kurz, R. Zirbel, S. Dietzel, B. Rinke, E. Schrock, M.R. Speicher, Mathieu, A. Jouch, P. Emmerich, H. Scherthan, T. Ried, C. Cremer and P. Lichter, Role of Chromosome territories in the functional compartmentalization of the cell nucleus. Cold Spring Harbor Symposium and Quantum Biology, 58, 777-792 (1993). 
29. M. Cremer, J. von Hase, T. Volm, A. Brero, G. Kreth, J. Walter, C. Fischer, I. Solovei, C. Cremer and T. Cremer, Non-random radial higher order chromatin arrangements in nuclei of diploid human cells. Chromosomes Research 9, 541-567 (2001).

30. C. Munkel, R. Eils, S. Dietzel, D. Zink, C. Mehring, G. Wedemann, T. Cremer and J. Langowski, Compartmentalization of interphase chromosomes observed in simulation and experiment. J. Mol. Biol. 285, 1053-1065 (1999).

31. D.E. Comings, The rationale for an ordered arrangement of chromatin in interphase nucleus. Am.J.Hum .Genet. 220, 440-448(1968).

32. A.E. Visser, F. Jaunin, S. Fakan and J.A, Aten, High resolution analysis of interphase chromosome domains. J. Cell Sci, 113, 2525-2593 (2000).

33. R. K. Sachs, G.van den Engh, B. Trask, H. Yokota and J.E. Hearst, A random walk giant-loop model for interphase chromosomes. Proc. Natl. Acad. Sci. 92, 2710-2714 (1995).

34. G. van den Engh, R. Sachs and B. Trask, Estimating genomic distances from DNA sequence location in cell nuclei by a random walk model. Science 257, 1410-1412 (1992).

35. H. Yokota, G. van den Engh, J.E. Hearst, R.K. Sachs and B.J. Trask, evidence for the organization of chromatin in megabase pair-sized loops arranged along a random walk path in the human $\mathrm{G}_{0} / \mathrm{G}_{1}$ interphase nucleus. J. Cell Biol. 130, 1239-1249 (1995).

36. N. Nagele, T. Freeman, L. McMorrow and H.Y. Lee, Precise spatial positioning of chromes during prometaphase: evidence for chromosomal order. Science 270,18311835 (1995).

37. D.C. Allison and A.L. Nestor, Evidence for a relatively random array of human chromosomes on the mitotic ring. J. Cell Biol. 145, 1-14 (1999).

38. A.C. Chandley, R.M. Speed and A.R. Leitch, Different distributions of homologous chromosomes in adult human sertoli cells and in lymphocytes signify nuclear differentiation. J. Cell Sci. 109, 773-776 (1996).

39. H.B. Sun, J. Shen and H. Yokota, Size-dependent positioning of human chromosome in nuclei. Biophys. J. 79, 184-190 (2000).

40. H. Ma, A. J. Siegel, and R. Berezney, Association of chromosome territories with nuclear matrix: disruption of human chromosome territories correlates with the release of a subset of nuclear matrix proteins. J. Cell Biol. 146, 531-541 (1999).

41. J.A. Croft, J. M. Bridger, S. Boyle, P. Perry, P. Teague, and W.A Brickmore, Differences in the localization and morphology of chromosomes in the human Nucleus. J. Cell Biol. 145, 1119-1131 (1999). 
42. S. Boyle, S. Gilchrist, J. M. Bridger, N. L. Mahy, J. A. Ellis and W. A. Bickmore, The spatial organization of human chromosomes within the nuclei of normal emerinmutant cells. Human Molecular Gentics. 10, 211-219 (2001).

43. 28. T. Inagaki, R.N. Hamm, E.T. Arakawa and L.R. Painter, Optical and dielectric properties of DNA in extreme ultraviolet. J. Chem. Phys. 61, 4246-4250(1974).

44. D. J. Brenner and M. Zaider, Modification of the theory of dual radiation action for attenuated fields. II. Application to the analysis of soft X-ray results. Radiat. Res. 99, 492-501 (1984).

45. D.J. Brenner, R.P. Bird, M. Zaider, P. Goldhagen, P.J. Kiiauga and H. H. Rossi, Inactivation of synchronized mammalian cells with low-energy x-rays-results and significance. Radiat. Res. 110, 413-427 (1987).

46. D. J. Brenner, On the probability of interaction between elementary radiation-induced chromosomal injuries. Rad. Envirn. Biophy. 27, 189-199 (1988).

47. B. Fouladi, C. A. Waldren, B. Rydberg and P. K. Cooper, Comparison of repair of DNA double-strand breaks in identical sequences in primary human fibroblast and immortal hamster-human hybrid cells harboring a single copy of chromosome 11. Radiat. Res. 153, 795-804 ( 2000).

48. M. Löbrich, P.K. Cooper and B. Rydberg, Joining of Correct and Incorrect DNA Ends at Double-Strand Breaks Produced by High-Linear Energy Transfer Radiation in Human Fibroblasts. Radiat.Res.150, 619-626 (1998).

49. T. Radivoyevitch, D. G. Hoel, P. J. Hahnfeldt, B. Rydberg and R.K. Sachs, Recent data obtained by pulsed-field gel electrophoresis suggest two types of double-strand breaks. Radiat. Res. 149, 52-58 ( 1998).

50. T. Radivoyevitch, D. G. Hoel, A. M. Chen and R. K. Sachs, Misrejoining of doublestrand breaks after X-irradiation : Relating moderate to very high doses by a Markov model .Radit.Res.149,59-67 (1998).

51. B.Rydberg, L.Heilbronn,W.R.Holley, M.Löbrich, C.Zeitlin, A. Chatterjee and P.K. Cooper, Spatial Distribution and Yield of DNA Double-Strand Breaks Induced by 3-7 MeV Helium Ions in Human Fibroblast. Radiat Res.158, 32-42 (2002).

52. J. N. Lucas, T. Tenjin, T. Straume, D. Pinkel, D. H. Moore, M. Lih and J.W. Gray, Rapid human chromosome aberration analysis using fluorescence in situ hybridization detection Int. J. Radiat. Biol. 56, 35-44 (1989).

53. P. Finnon, D.C. Lloyd and A.A. Edwards, Fluorescence in situ hybridization detection of chromosomal aberrations in human lymphocytes: applicability to biological dosimetry. Int. J. Radiat. Biol.,68, 429-435 (1995).

54. D.C. Lloyd,A.A. Edwards and J.S. Prosser, Chromosome aberrations induced in human lymphocytes by in vitro acute $\mathrm{X}$ and gamma radiation. Radiat. Prot. Dosim., 15, 83-88 (1986).

55. A.A Edwards, The use of chromosomal aberrations in human lymphocytes for 
biological dosimetry. Radiat. Res.,148,539-544 (1997).

56. J. D. Tucker, K. J. Sorensen, Cathy S. Chu, D.O. Nelson, M.J.Ramsey, C. Urlando and J.A. Heddle, The accumulation of chromosome aberrations and DLB-L. in ice with highly fractionated exposure to gamma radiation. Mut. Res. 400, 321335 (1998).

57. A. L. Brooks, B. Shiping, K. Rithidech, L.A. Couch and L.A. Braby, Relative Effectiveness of HZE iron-56 particles for the induction of cytogentic damage in vivo. Radiat. Res. 155, 353-359 (2001)

58.R. J. Simpson and J. R. K. Savage, Dose-response curves for simple and complex chromosome aberrations induced by x-rays and detected using fluorescence in situ hybridization. Int. J. Radiat. Biol. 69, 4299-436 (1996).

Table. I. Pairwise parameters of the model for which theoretical results on interchromosomal aberration frequencies as a function of dose are in good agreement with all the experimental data.

\begin{tabular}{|c|c|}
\hline $\begin{array}{c}\text { Overlap Parameter } \\
\Omega(\mu \mathrm{m})\end{array}$ & $\begin{array}{c}\text { Range Parameter } \\
\sigma(\mu \mathrm{m})\end{array}$ \\
\hline & \\
0.300 & 0.525 \\
0.675 & 0.500 \\
1.000 & 0.400 \\
\hline
\end{tabular}

Figure Legends

Figure 1 a Random polymer model of chromosome 4 projected onto x-y plane. Each plotted segment represents a section of $30 \mathrm{~nm}$ chromation fiber consisting of approximately $7.7 \mathrm{kbp}$ organized in a zig-zag ribbon configuration. The line between the 
two solid circles corresponds to a projected distance of $1.6 \mu \mathrm{m}$ and a separation of about $30 \mathrm{Mbp}$.

Figure 1 b Mean-square, projected distances between points on chromosome 4 vs. genomic separation in Mbp for the theoretical model (solid curve) and experimental data of Yokota et al. (35).

Figure 2. Example of 46 randomly placed chromosomes packed within a $6.5 \mu \mathrm{m}$ radius sphere using a maximum overlap $\Omega=0.675 \mu \mathrm{m}$ and a maximum gap $\Gamma=0.0$. These parameters result in every chromosome touching or overlapping at least one of its neighbors. Spheres of the same color correspond to pairs of autosomes within the nucleus. A and B are two orthogonal views of the same nucleus. Note that the $6.5 \mu \mathrm{m}$ is a limiting radius used in packing the chromosomes inside a nuclear volume and is larger than the effective radius of the nucleus (closer to $5.5 \mu \mathrm{m}$ ) if drawn to touch the chromosome surfaces.

Figure 3. Shown here are examples of the simplest chromosome aberrations which result from the rejoining algorithm. Consider the situation where there are double strand breaks, on two nearby chromosomes as in (a) and (b). In this case the relative probabilities of misrejoining to form either a reciprocal translocation or a dicentric will be equal because, for a given pair of breaks, the distances between various combinations of ends in (a) and (b) are the same. The configuration (f) illustrates the simplest complex rearrangement (insertion) involving three double strand breaks and two chromosomes. Besides this simple case we have detected many complicated complex aberrations, which cannot be characterized by the simple cases represented in this figure. 
Figure 4. Three typical trial calculations showing the distribution of DSBs indicated by open circles. The cases involve not only different LET values but also distinct track structures represented by (a) electrons (similar to X-ray irradiation),( b) $1 \mathrm{MeV} / \mathrm{n}$ helium particles and (c) $1000 \mathrm{MeV} / \mathrm{n}$ iron particles. In each case the average dose is $1 \mathrm{~Gy}$.

Figure 5. Theoretical yields of total inter-chromosomal rejoinings per cell as a function of dose for low-LET electron irradiation for three values of the proximity range parameter $\sigma(400,500$ or $600 \mathrm{~nm})$. Results are for all exchanges, including those with DNA segments too small to be detected cytogenetically. The model nucleus is the same for each case and had an overlap parameter $\_0.675 \_\mathrm{m}$.

Figure 6. Theoretical yields of inter-chromosomal rejoinings per cell as a function of dose for low LET electron irradiation and resolution limits of 1 bp, $1 \mathrm{Mbp}, 10 \mathrm{Mbp}$ and $20 \mathrm{Mbp}$. The overlap parameter $\Omega=0.675 \mu \mathrm{m}$ and the proximity range parameter $\sigma=0.5$ $\mu \mathrm{m}$.

Figure 7. A comparison of theoretical yields based on the model at two different resolutions (1 Mbp and $20 \mathrm{Mbp}$ ) with experimental data reported in the literature. Since translocation and dicentric yields in the model are the same, comparisons have been made with translocation measurements of Lucas et al. (52) and Finnon et al. (53) and dicentric measurements of Lloyd et al. (54) and Edwards (55). To represent the measurements of Lloyd et al. and Edwards, we have used their linear-quadratic expressions, (0.005 + $\left.0.036 \mathrm{D}+0.067 \mathrm{D}^{2}\right)$ and $\left(0.018 \mathrm{D}+0.060 \mathrm{D}^{2}\right)$, respectively. For the most part the experimental data compared here are contained within the theoretical curves corresponding to the two resolution limits.

Figure 8. Comparison of the experimental data for translocation frequencies per fraction from Tucker et al. (56) with theoretical results for resolution limits of $1 \mathrm{Mbp}$ and 10 Mbp. The experimental data are from the whole body irradiation of mice with low doses 
per fraction ( $1-8 \mathrm{cGy}$ ) of $\gamma$ rays. The theoretical calculations use a model of the mouse nucleus containing 40 chromosomes. The overlap parameter $\Omega=0.675 \mathrm{~nm}$ and proximity parameter $\sigma=0.5 \mu \mathrm{m}$ were the same as in the human calculations. Considering the scatter in the experimental points it is not possible to choose between the two resolution limits.

Figure 9. The number of total complex exchanges in human lymphocytes per cell as a function of dose. The experimental data of Loucas and Cornforth (5) generally lie between the two theoretical curves with resolution limits of $10 \mathrm{Mbp}$ (upper curve) and 20 Mbp (lower curve). In the theoretical calculations, the proximity range parameter $\sigma=.5$ $\mu \mathrm{m}$ and the overlap parameter $\Omega=0.675 \mu \mathrm{m}$.

Figure 10. The calculated yield of total inter-chromosomal rejoinings as a function of dose for $1000 \mathrm{MeV} / \mathrm{n}$ iron particles. No experimental data is available for comparison, therefore, results should be considered as predictions of the model. The average number of particles through a cell nucleus is indicated at each dose value. The range parameter $\sigma=0.5 \mu \mathrm{m}$ and overlap parameter $\Omega=0.675 \mu \mathrm{m}$ were the same as in the low-LET calculations. Our theoretical results for high-LET heavy ions show a linear dose dependence instead of the linear-quadratic dose dependence evident for low-LET radiation. A linear dose dependence for $1000 \mathrm{MeV} / \mathrm{n}$ iron particles has been reported by Brooks et al. (57) for chromatid type aberrations. 

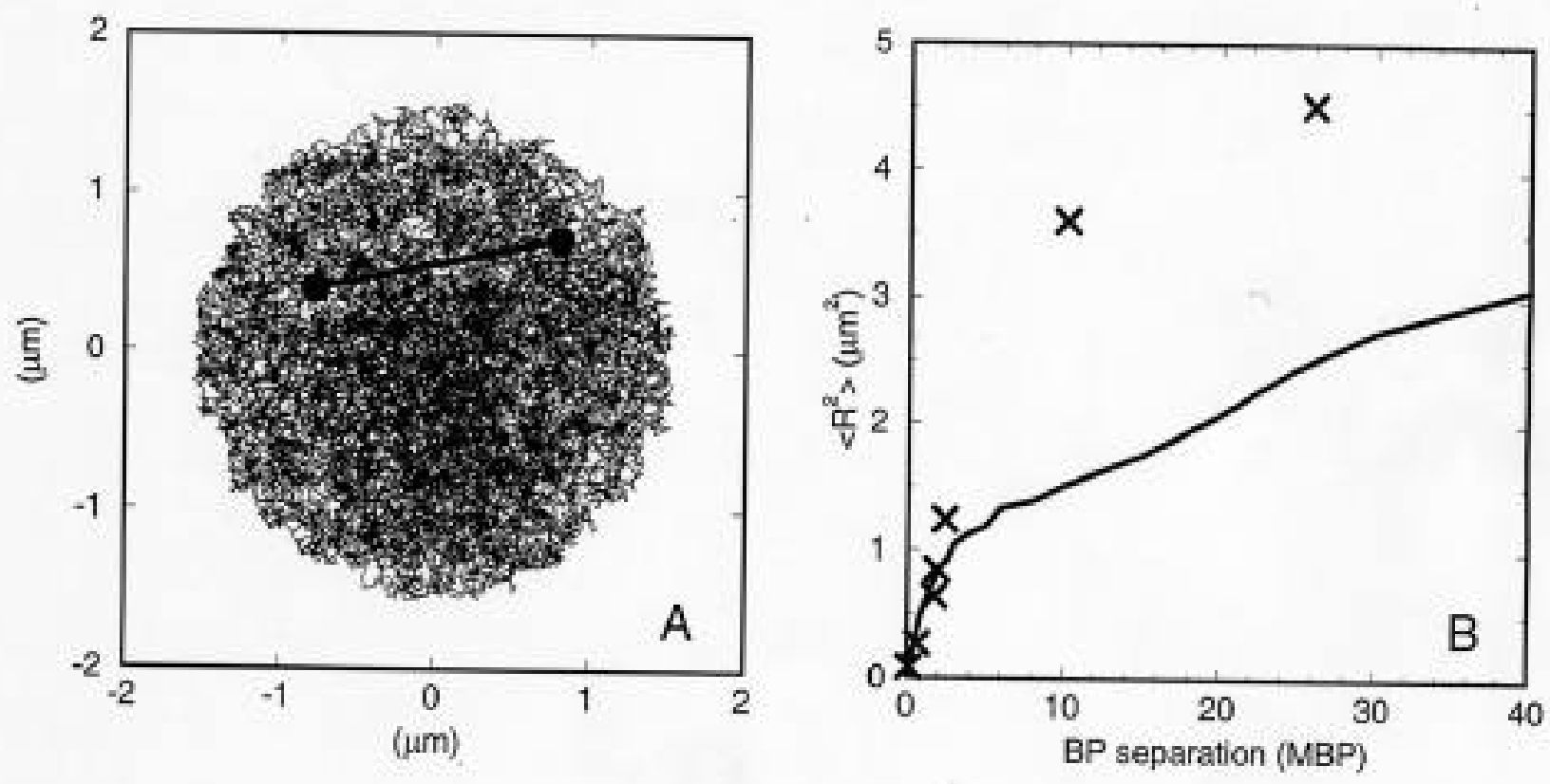


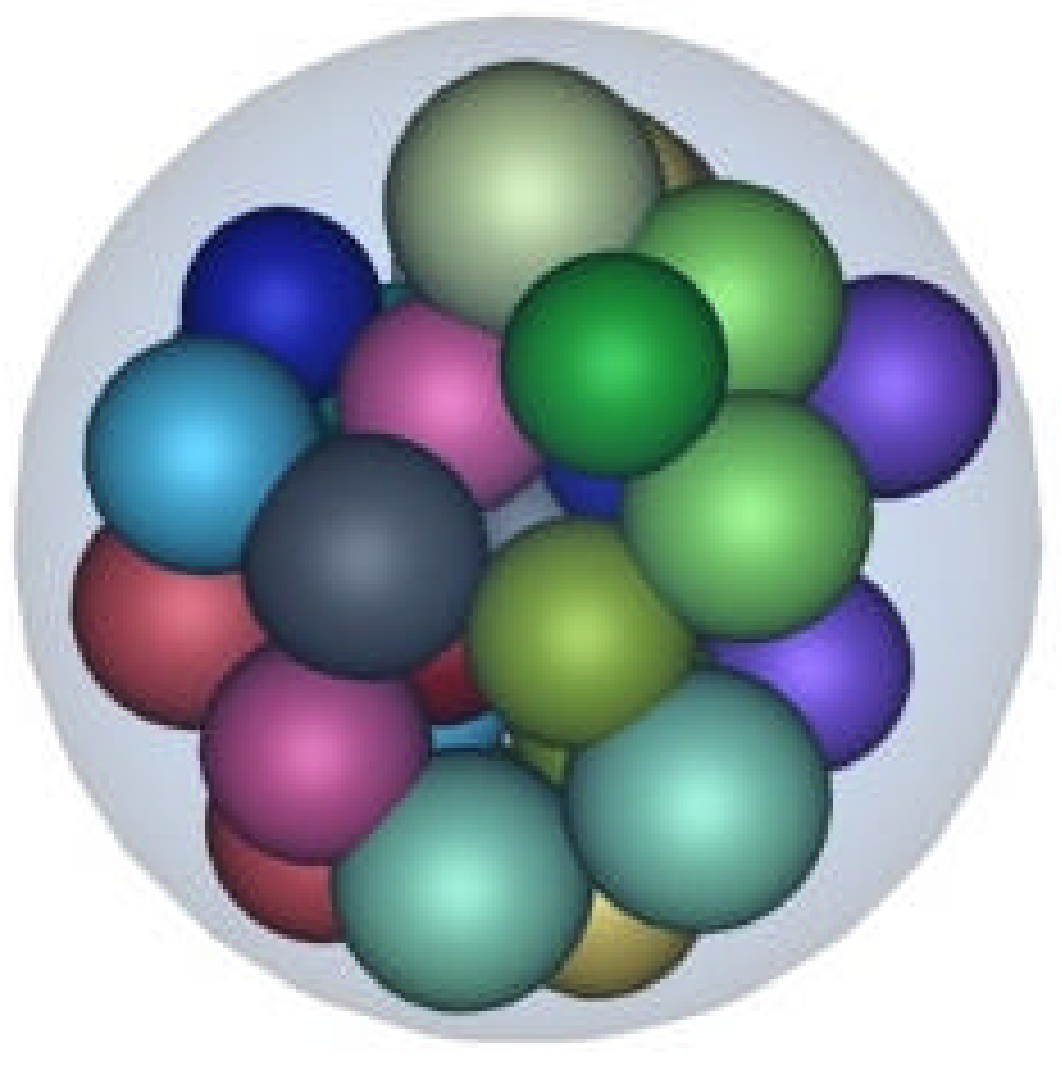

A

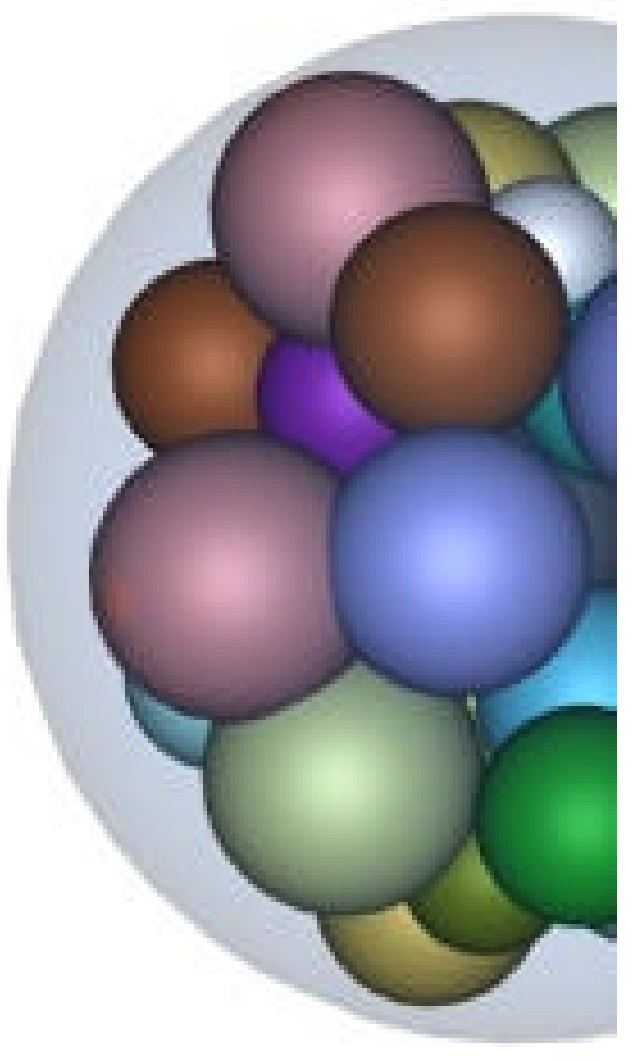

B 

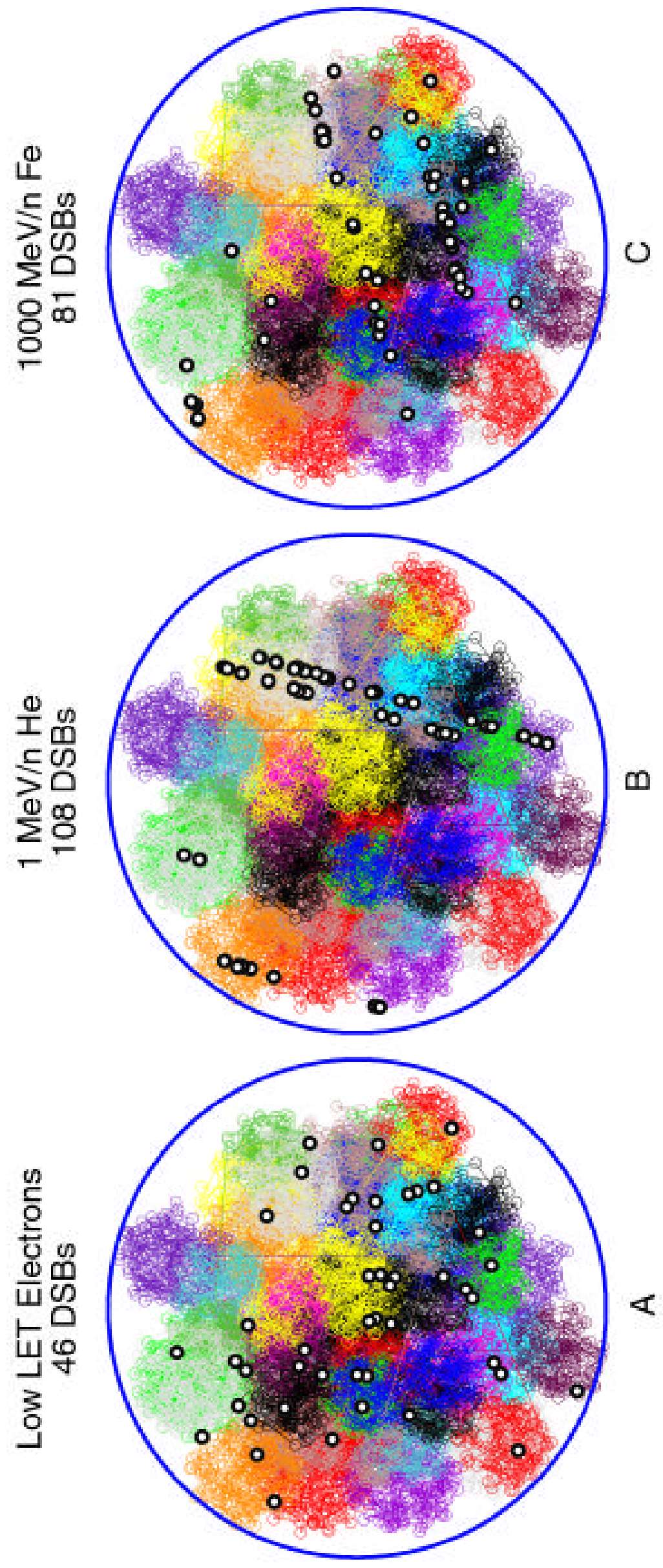


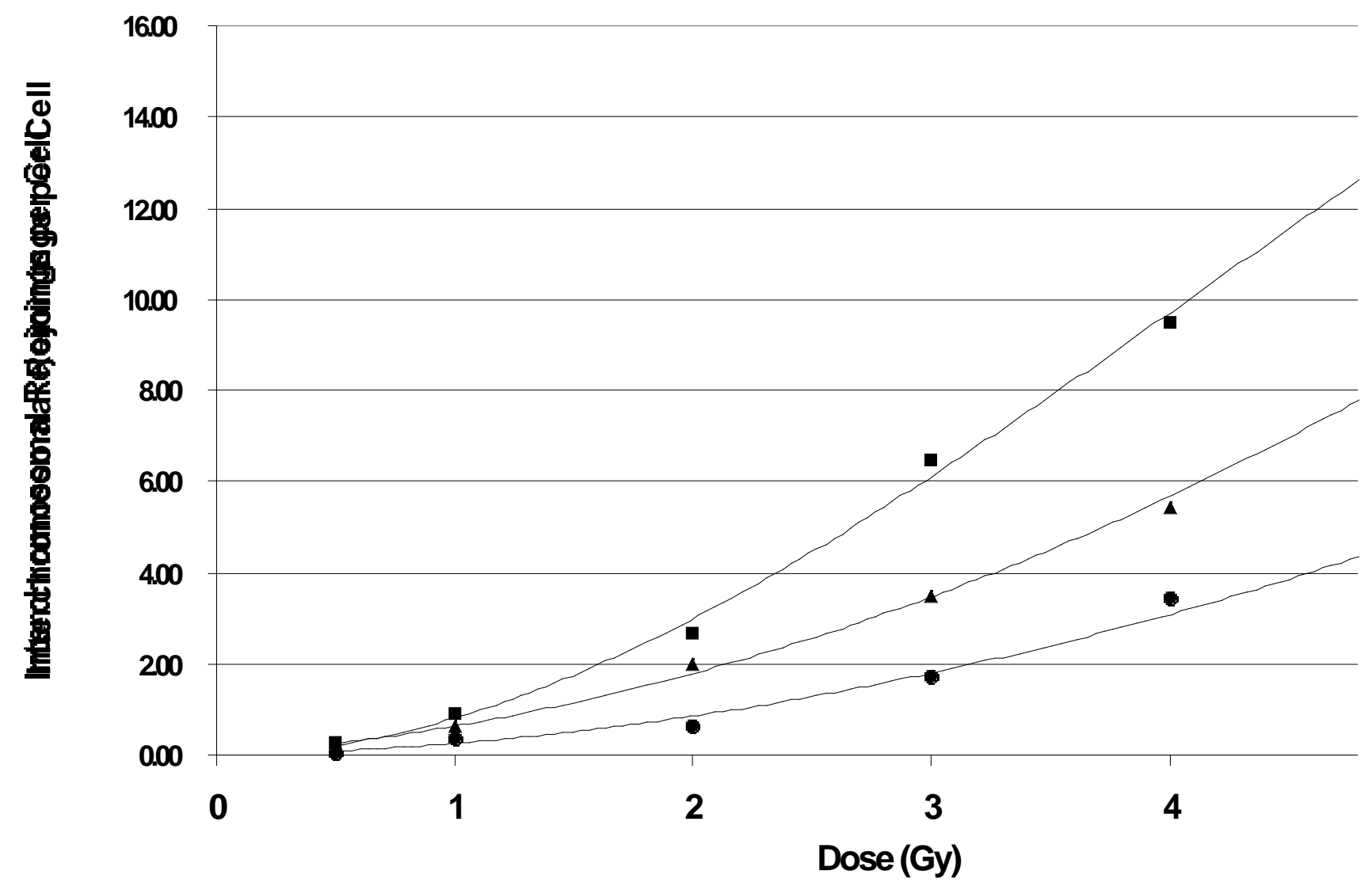





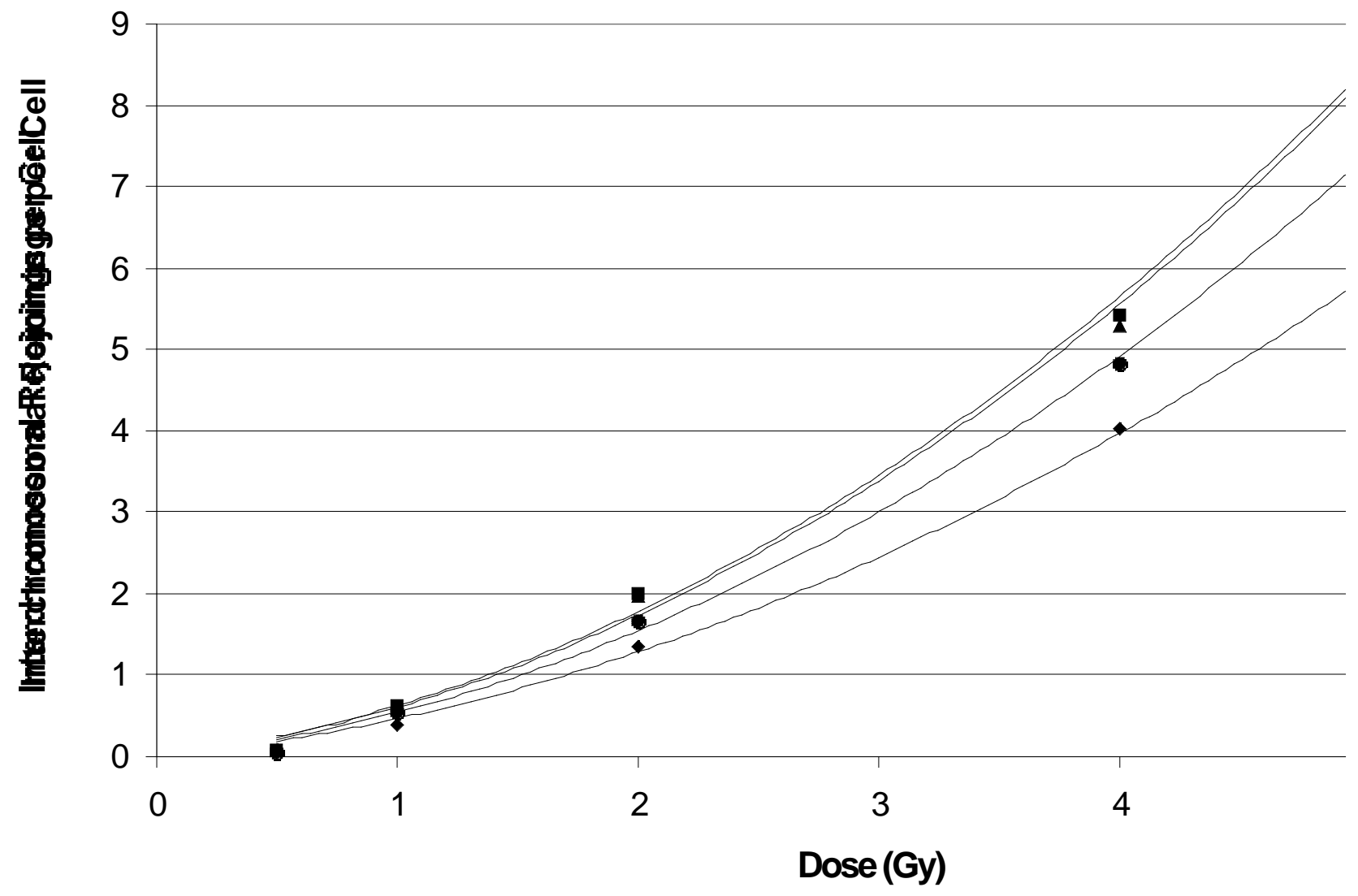





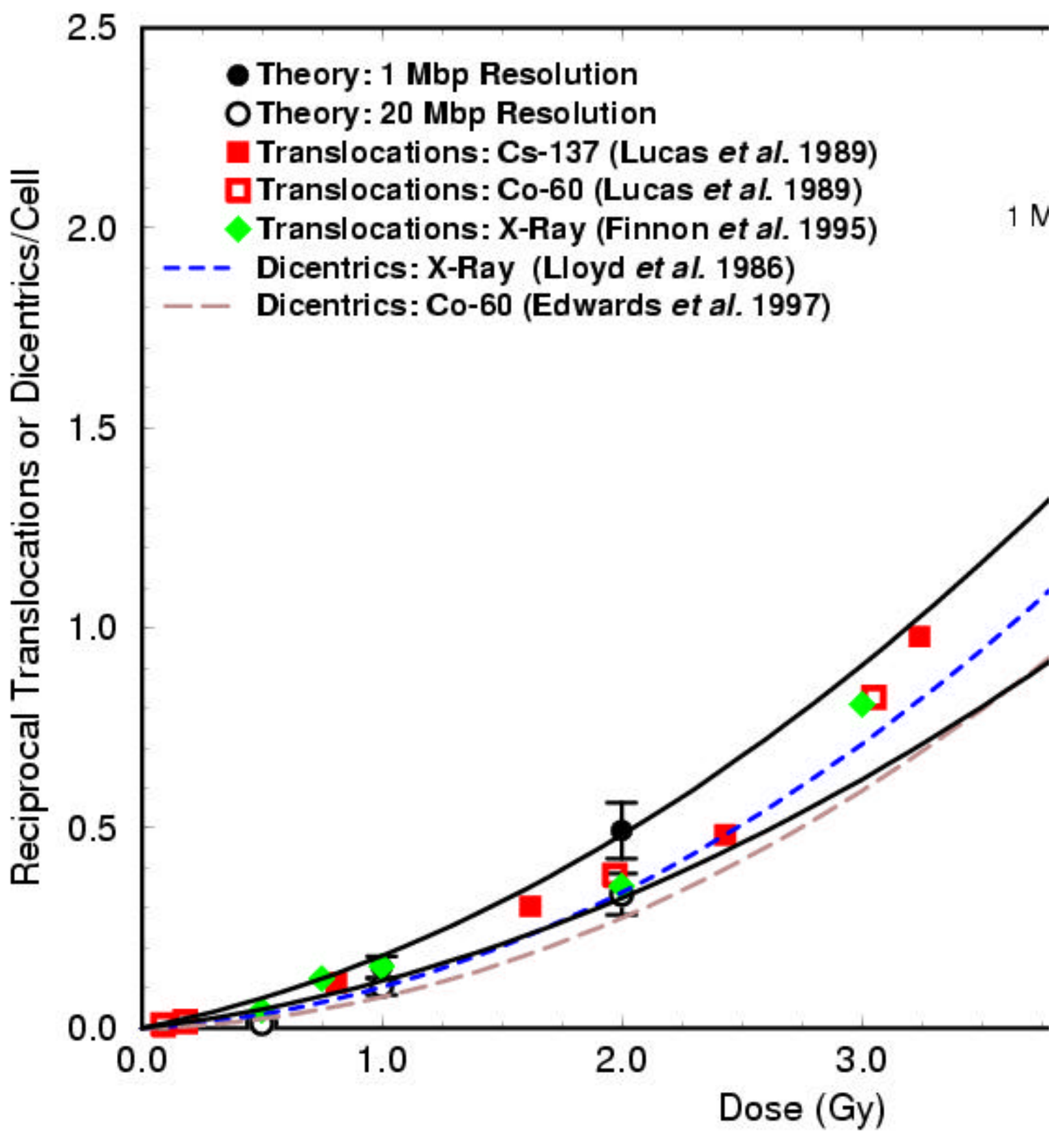




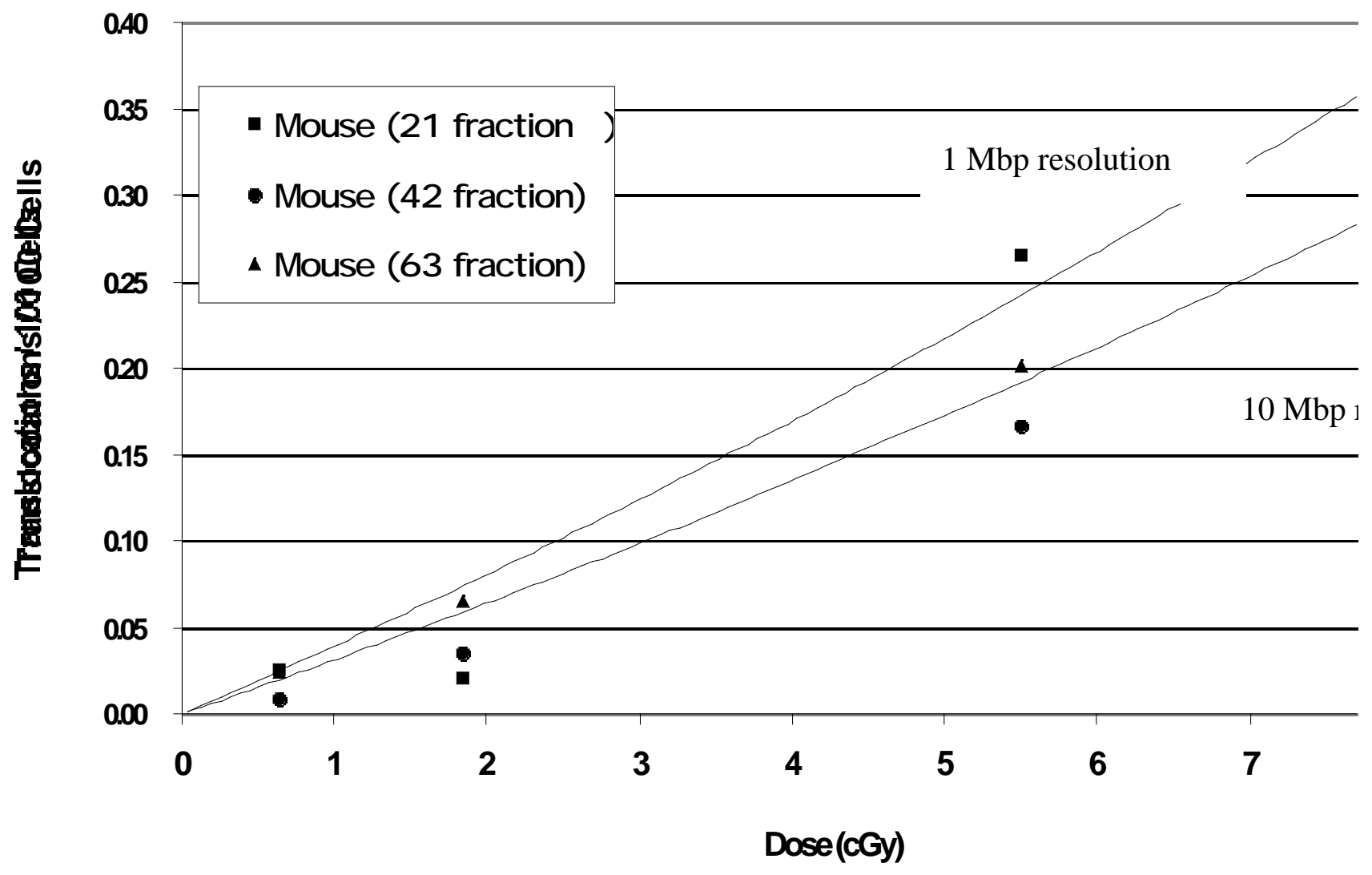




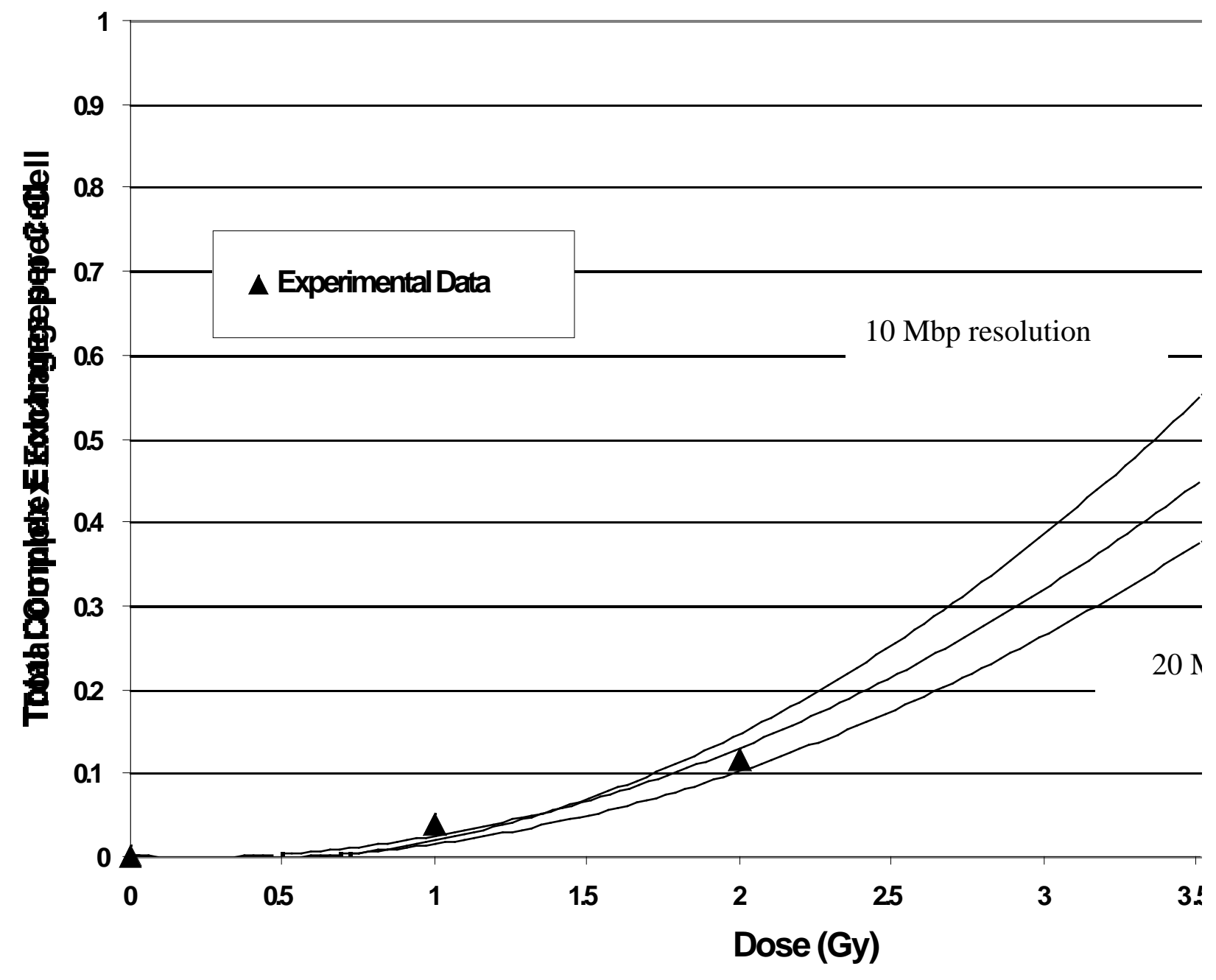




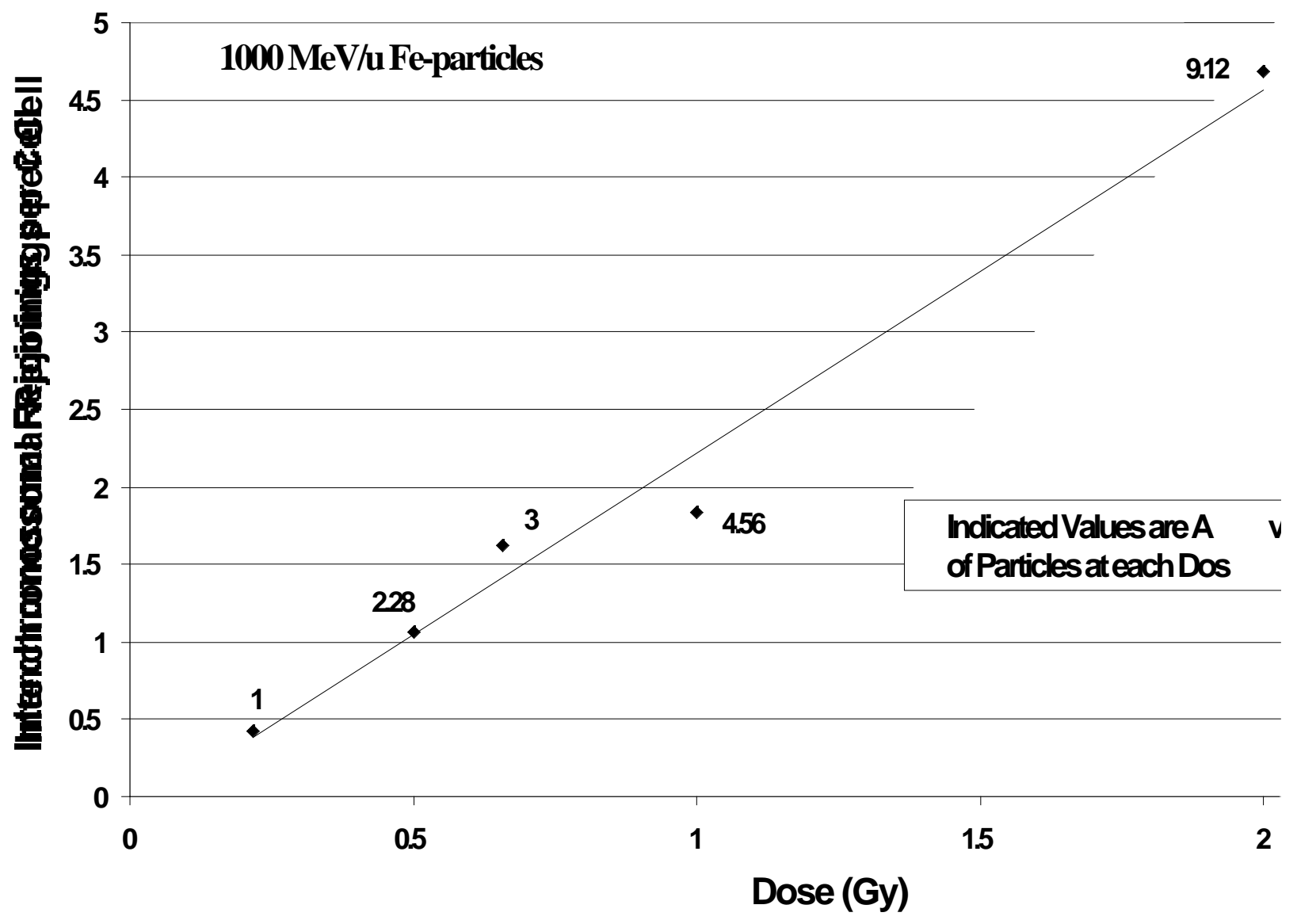

